

https://doi.org/10.31533/pubvet.v15n11a970.1-8

\title{
Edema pulmonar cardiogênico em cadela: Relato de caso
}

\author{
Hellen Christina Cordeiro da Rocha ${ }^{1 *} \bullet$, Anny Cristina Veras Leite ${ }^{2} \bullet$, Mirella Oliveira Zanini ${ }^{3 \bullet}$ \\ ${ }^{I}$ Discente do curso de medicina veterinária do Centro Universitário do Norte (UNINORTE), Manaus - AM. Brasil. \\ ${ }^{2}$ Prof ${ }^{a}$ Mestre do UNINORTE, Departamento de Medicina Veterinária. Manaus - AM, Brasil. \\ ${ }^{3}$ Médica Veterinária. Manaus - AM, Brasil. \\ * Autor para correspondência, E-mail: Christina_rochahc@hotmail.com
}

Resumo. O edema pulmonar consiste no acúmulo de líquido dentro do parênquima pulmonar. De acordo com a fisiologia pode ser de classificado como cardiogênico e não cardiogênico. Os sinais clínicos envolvem dispneia, tosse, taquipneia e, alteração dos sons normais na ausculta pulmonar. No presente relato, foi descrito um caso de um canino fêmea, SRD, 10 anos que inicialmente foi a clínica por quadros de tosse. Porém, após alguns dias voltou na emergência da clínica apresentando quadros de dispneia. Após estabilização do paciente, foram realizados exames de radiografia, ultrassonografia e eletrocardiograma que constataram edema pulmonar cardiogênico decorrente de cardiopatia esquerda. Após a abordagem emergencial, a paciente permaneceu internada por um período de 24 horas, sendo liberada após este. O tratamento crônico da cardiopatia foi prescrito e solicitado acompanhamento com cardiologista a cada 6 meses. Apesar de se tratar de uma urgência veterinária, quando tratados corretamente e então solucionar a causa base, o prognóstico tende a ser favorável.

Palavras-chave: Cardiopatia, edema pulmonar, emergência veterinária

\section{Cardiogenic pulmonary edema in a female canine: Case report}

Abstract. Pulmonary edema is the accumulation of fluid within the lung parenchyma. According to its physiology it can be classified as cardiogenic and non-cardiogenic. The clinical signs involve dyspnea, cough, tachypnea, and alteration of the normal sounds on pulmonary auscultation. In this report, was described a case of a female canine, SRD, 10 years old that initially went to the clinic for coughing. However, after a few days it returned to the emergency room presenting dyspnea. After stabilizing the patient, radiography, ultrasonography, and electrocardiogram were performed, which revealed cardiogenic pulmonary edema due to left heart disease. After the emergency approach, the patient remained hospitalized for a period of 24 hours, after which it was released. Chronic treatment of the heart disease was prescribed and follow-up with a cardiologist every 6 months was requested. Although this is a veterinary emergency, when treated correctly and then solving the underlying cause, the prognosis tends to be favorable.

Keywords: Heart disease, pulmonary edema, veterinary emergency

\section{Edema pulmonar cardiogénico en una perra: Informe de caso}

Resumen. El edema pulmonar es la acumulación de líquido dentro del parénquima pulmonar. Según su fisiología, puede clasificarse como cardiogénica o no cardiogénica. Los signos clínicos incluyen disnea, tos, taquipnea y alteración de los sonidos normales en la auscultación pulmonar. En el presente informe, se describió un caso de un canino hembra, mestizo, de 10 años de edad que inicialmente acudió a la clínica debido a la tos. Sin embargo, al cabo de unos días volvió a la sala de urgencias de la clínica presentando 
disnea. Tras estabilizar a la paciente, se realizaron radiografía, ecografía y electrocardiograma, que mostraron un edema pulmonar cardiogénico debido a una cardiopatía izquierda. Tras el abordaje de urgencia, el paciente permaneció hospitalizado durante un periodo de 24 horas, siendo dado de alta tras este periodo. Se prescribió el tratamiento crónico de la enfermedad cardíaca y se solicitó un seguimiento con un cardiólogo cada 6 meses. Aunque se trata de una urgencia veterinaria, cuando se trata correctamente y se resuelve la causa de base, el pronóstico suele ser favorable.

Palabras clave: enfermedad cardíaca, edema pulmonar, emergencia veterinaria

\section{Introdução}

O edema pulmonar agudo em cães frequentemente é resultado de afecção valvar degenerativa crônica e cardiomiopatia dilatada. Em gatos, geralmente é consequência de insuficiência cardíaca diastólica associada a cardiomiopatia hipertrófica ou restritiva (Feldman, 1997; Jericó et al., 2015). Segundo Gaynor \& Muir (2009), outras cardiomiopatias envolvidas são endomiocardite, arritimias severas e defeito do septo ventricular. Todavia, a insuficiência do coração esquerdo, frequentemente decorrente da insuficiência de mitral ou de bombeamento é a causa mais comum desta patologia (Ettinger et al., 2002).

Segundo Glaus et al. (2010), o movimento fisiológico do fluido através da membrana vascular para o tecido circundante depende de alguns fatores, dentre eles a permeabilidade alveolocapilar, o gradiente de pressão oncótica, o gradiente de pressão hidrostática e a drenagem linfática. Quando há um desequilíbrio desses fatores, pode-se desenvolver o edema. Segundo Castro (2003), o acúmulo de líquido no pulmão independente do mecanismo desencadeador pode ser dividido em três estágios, conforme tabela 1.

Tabela 1. Estágios de evolução do edema pulmonar

\begin{tabular}{lclll}
\hline \multicolumn{3}{c}{ Primeiro estágio } & Segundo estágio & Terceiro estágio \\
\hline Aumento do fluxo de líquidos dos & Acúmulo de líquido no interstício em & Aumentos adicionais do volume no \\
capilares para o interstício, & razão de o volume que é filtrado pelos & interstício que levam à distensão dos \\
paralelamente $\quad$ ao $\quad$ aumento & capilares ultrapassar a capacidade & septos interalveolares e consequente \\
compensatório da drenagem linfática. & máxima de drenagem linfática. & inundação dos alvéolos. \\
\hline
\end{tabular}

Fonte: Bitencourt et al. (2017).

A presença de líquido nos alvéolos dificulta a ventilação das áreas pulmonares afetadas levando o animal a hipoxemia (Ettinger et al., 2002; Feldman, 1997). Segundo Castro (2003) e Bitencourt et al. (2017), a distribuição do edema não é homogênea, pois em regiões com declive a pressão intravascular é maior. A formação de edema progride do espaço intersticial para o espaço alveolar, com sinais que variam de taquipneia a dispneia grave, de acordo com o grau de insuficiência cardíaca em que o paciente apresenta (Jericó et al., 2015). Dependendo do grau do edema, os pacientes podem apresentar tosse, taquipneia, dispneia ou, em casos mais graves, distrição respiratória acentuada (Bitencourt et al, 2017). Os sons respiratórios geralmente se encontram altos e ásperos (dispneia inspiratória) e o prejuízo da ventilação-perfusão resulta em hipoxemia (Jericó et al., 2015). Segundo Bitencourt et al. (2017), no edema cardiogênico, além das alterações pulmonares, há evidências de alterações hemodinâmicas, como hipotensão, hipertensão e arritmias, compatíveis com cardiopatia e/ou choque cardiogênico. À ausculta cardíaca, encontra-se ritmo de galope, sopro cardíaco e, dependendo da causa primária, arritmias.

A abordagem inicial do paciente instável deve ser direcionada a identificar os achados-chave do histórico e exame físico que caracterizem esse paciente como portador de edema pulmonar agudo emergencial, como: sopro cardíaco ou ritmo de galope, arritmia cardíaca, histórico de cardiopatia, sinais e sintomas clínicos (Jericó et al., 2015).

O diagnóstico inicial é obtido por ausculta torácica e sintomas. A radiografia torácica deve ser realizada quando o paciente estiver mais estável e for capaz de tolerar o procedimento Jericó et al. (2015). A distribuição do padrão alveolar pode auxiliar na identificação de um edema pulmonar cardiogênico ou não cardiogênico, no entanto, ambas as situações podem apresentar um padrão alveolar difuso (Bitencourt et al. 2017). 
Segundo Jericó et al. (2015), o tratamento deve ser sintomático para estabilização antes dos exames, buscando aliviar sinais de congestão, melhorar a função cardíaca e promover a perfusão tecidual e reduzir o nível de estresse do paciente. A oxigenoterapia deve ser iniciada rapidamente em pacientes com angústia respiratória. $\mathrm{O}$ esforço respiratório leva a um aumento do gasto energético e pode contribuir para a hipertermia e a insuficiência respiratória, exacerbando os sinais clínicos. Em caso de animais muito agitados com a dispneia e se houver estabilidade hemodinâmica, pode-se sedar levemente esses pacientes para facilitar o manejo e amenizar a fadiga respiratória (Bitencourt et al, 2017). Recomenda-se o uso de tranquilizantes, como a acepromazina em doses baixas $(0,01-0,02$ $\mathrm{mg} / \mathrm{kg})$, podendo esta ser associada a um opioide para potencializar o efeito, como o butorfanol $(0,1 \mathrm{a}$ $0,4 \mathrm{mg} / \mathrm{kg}$ ) ou a meperidina ( 3 a $5 \mathrm{mg} / \mathrm{kg}$ ). A necessidade da sedação deve ser ponderada e o paciente deve ser monitorado para evitar depressão respiratória e cardíaca (Rozanski \& Rush, 2009; Rozanski, 2013).

O oxigênio pode ser fornecido por meio de uma variedade de opções, incluindo fluxo flow-by, que utiliza tubo de silicone de grosso calibre diretamente conectado ao cilindro de oxigênio entre 1 e $3 \mathrm{~cm}$ de distância da narina do animal (Jericó et al., 2015), máscara facial, oxigênio por sonda nasal unilateral ou bilateral, colar elisabetano adaptado com envoltório de celofane ou papel filme, gaiola de oxigênio e intubação (Bitencourt et al., 2017).

Segundo Bucceroni (2021), o ar ambiente possui uma estimativa da fração inspirada de oxigênio (FiO2) de $21 \%$. Na tabela 2 observa-se a estimativa da Fração Inspirada de Oxigênio (FiO2) de acordo com o fluxo de oxigênio (litros/minuto) e as diferentes técnicas de administração.

Tabela 2. Estimativa da FiO2 de acordo com fluxo de oxigênio (L/min) e as diferentes técnicas de administração

\begin{tabular}{lcr}
\hline Técnica de administração & Fluxo de O2 (L/min) & FiO2 estimada (\%) \\
\hline Máscara facial & $2-5$ & $40-50$ \\
Colar elizabetano adaptado & $1-5$ & $30-50$ \\
Sonda nasal unilateral & $<2$ & $40-50$ \\
Sonda nasal bilateral & $<2$ para cada narina & $40-50$ \\
Gaiola de oxigênio & O necessário para manter a FiO2 entre 40- $60 \%$ & $21-60$ \\
Ventilação mecânica & $1-2$ & $21-100$ \\
Flow - By & $2-5$ & $25-40$ \\
\hline
\end{tabular}

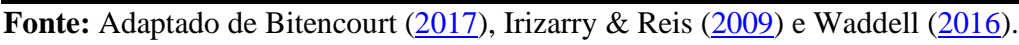

Segundo Bitencourt et al. (2017), os diuréticos são utilizados na terapia do edema pulmonar, tanto cardiogênico como não cardiogênico, com o objetivo de reduzir o volume de fluido circulante e a précarga e, com isso, melhorar o edema pulmonar. A furosemida é o diurético de escolha e deve ser administrada diretamente por via intravenosa, sempre que possível, ou intramuscular quando houver suspeita de insuficiência cardíaca, na dose inicial de 2 a $4 \mathrm{mg} / \mathrm{kg}$, podendo chegar a $8 \mathrm{mg} / \mathrm{kg}$ e ser repetida de acordo com a necessidade, com base na frequência respiratória em repouso (Jericó et al., 2015). O uso de vasodilatadores, como a nitroglicerina e o nitroprussiato, é recomendado. O nitroprussiato, um potente vasodilatador arterial e venoso, é utilizado por infusão contínua, em equipo ou seringa fotossensível, usualmente na taxa de 0,5 a $2 \mu \mathrm{g} / \mathrm{kg} / \mathrm{min}$, mas podendo chegar até 5 $\mu \mathrm{g} / \mathrm{kg} / \mathrm{min}$. A monitoração constante da pressão arterial, de preferência de forma invasiva, é fundamental para evitar hipotensão grave promovida pelo uso dos vasodilatadores (Bitencourt et al, 2017).

A nitroglicerina está disponível na forma de adesivos transdérmicos que podem ser aplicados, após tricotomia, sobre a pele da pina ou da região inguinal, aquela que estiver mais aquecida, indicando melhor perfusão. A dose é $1 / 8$ de um adesivo de $5 \mathrm{mg}$ para cães pequenos e $1 / 2$ a 1 adesivo para cães grandes, 1 vez ao dia, por 2 a 3 dias (Jericó et al., 2015).

Segundo Bitencourt et al. (2017), em pacientes com débito cardíaco e pressão arterial reduzidos, em infusão ou não de vasodilatadores, recomenda-se o uso de inotrópicos. A dobutamina fornece suporte inotrópico na falência miocárdia, cão 5 a $20 \mu \mathrm{g} / \mathrm{kg} / \mathrm{min}$, gato 0,5 a $2 \mu \mathrm{g} / \mathrm{kg} / \mathrm{min}$ (Jericó et al., 2015). A hidralazina promove arteriodilatação e pode ser usada para reduzir a fração regurgitante da degeneração mixomatosa da valva mitral, na dose de 0,75 a $3 \mathrm{mg} / \mathrm{kg}$ por via oral, para se obter pressão arterial sistêmica sistólica entre 90 e $110 \mathrm{mmHg}$ (Jericó et al., 2015). O prognóstico de um animal com 
edema pulmonar depende da gravidade do edema, da resposta à terapêutica empregada (oxigenoterapia e farmacológica) e da doença primária (Bitencourt et al, 2017).

\section{Relato de caso}

Foi admitida na clínica veterinária Buldogue Verde um canino, fêmea, sem raça definida (SRD), de 10 anos, pesando $13,8 \mathrm{~kg}$. O tutor relatava que o animal, no dia anterior, apresentou quadros de tosse e "roncado", mas que já havia apresentado antes, porém em menor frequência. Segundo o tutor, o animal apresentava normoquesia, normofagia e normodipsia.

Ao exame clínico o animal apresentava parâmetros normais e um leve sopro na auscultação, porém sem quadros de tosse durante o exame. Foram realizados os exames de hemograma e bioquímico onde todos estavam sem alteração, incluindo as enzimas renais e hepáticas. Foi solicitado radiografia de tórax e encaminhado para consulta com cardiologista para realização de eletrocardiograma.

Após quatro dias da última consulta, o animal retornou na emergência da clínica apresentando quadro de dispneia, na auscultação estavam presentes ruídos característicos de estertor pulmonar. Foi iniciado o suporte de oxigenioterapia, aplicado furosemida na dose de $3 \mathrm{mg} / \mathrm{kg}$ por via endovenosa e dexametasona na dose $0,5 \mathrm{mg} /$ animal por via subcutânea.

Durante o período de internação a paciente continuou com a oxigenioterapia alternando com nebulização com $10 \mathrm{ml}$ de soro fisiológico com cinco gotas de bisolvon QID. Após a estabilização foram efetivados exame de radiografia ((Figuras 1-2)e , ultrassonografia e eletrocardiograma que não haviam sido realizados anteriormente pelo tutor.
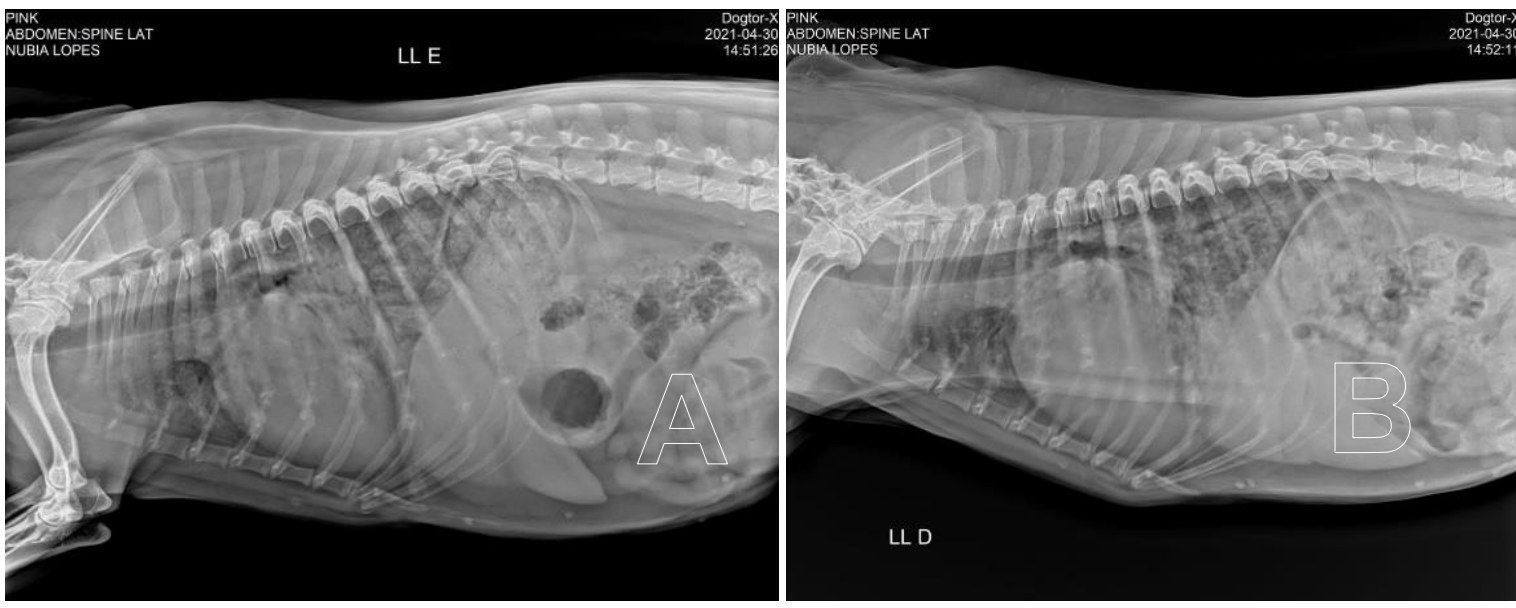

Figura 1. Radiografia na projeção latero-lateral esquerdo (A) e direito (B) do paciente.



Figura 2. Radiografia na projeção ventro-dorsal do paciente. 
Tabela 3. Laudo do exame de radiografia do paciente.

\section{Descrição}

Importante opacificação dos lobos pulmonares caudais de padrão alveolar e tendendo ao alveolar em lobo médio.

Silhueta cardíaca com aumento de suas dimensões em correspondência ao eixo ápicobasilar, promovendo desvio dorsal do trajeto traqueal.

Lúmen traqueal preservado.

Grandes vasos com calibre e trajeto preservados.

Discreta quantidade de conteúdo gasoso no lúmen esofágico - aerofagia.

Sem evidências de derrame pleural ou linfonodomegalia mediastinal detectáveis ao exame radiográfico.

Calcificação das cartilagens costais e osteoartrose das articulações costocondrais (processo senil).

Silhueta hepática ultrapassando os limites do rebordo costal.

Conteúdo gasoso e heterogêneo com característica de ingesta preenchendo a cavidade gástrica.

\section{Impressão diagnóstica}

Edema pulmonar cardiogênico importante.

Cardiomegalia esquerda.

Hepatomegalia.

O paciente realizou exame de ultrassonografia onde também foi evidenciada hepatomegalia e aumento da silhueta cardíaca, porém demais órgãos sem alterações na motilidade ou morfologia (Figura 3).
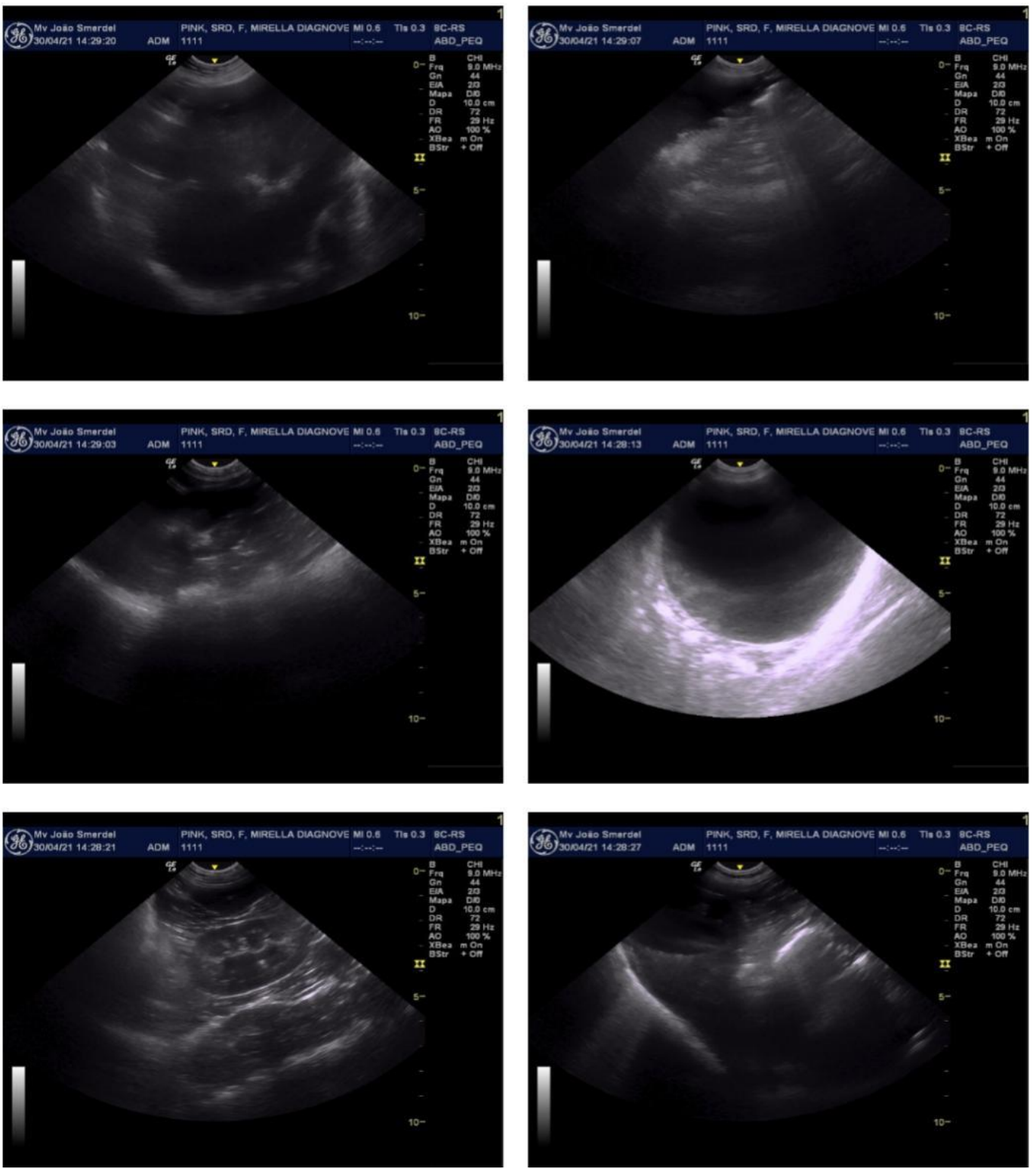

Figura 3. Imagens do exame ultrassonográfico da paciente. 




Figura 4. Eletrocardiograma da paciente.

Tabela 4. Laudo do exame de eletrocardiograma

\begin{tabular}{lll}
\hline Parâmetros observados & Parâmetros observados & Parâmetros observados \\
\hline Eixo QRS: $79.82^{\circ}$ & Amplitude de P: $0.44 \mathrm{mV}$ & Amplitude de R: $2.27 \mathrm{mV}$ \\
Eixo P: $77.25^{\circ}$ & Segmento PR: $26 \mathrm{~ms}$ & Intervalo PR: $76 \mathrm{~ms}$ \\
Duração de T: $80 \mathrm{~ms}$ & Amplitude de T: $-0.47 \mathrm{mV}$ & Segmento ST: $58 \mathrm{~ms}$ \\
Amplitude de Q: $-0.19 \mathrm{mV}$ & Intervalo QT: $216 \mathrm{~ms}$ & Duração de P: $50 \mathrm{~ms}$ \\
Duração de QRS: $78 \mathrm{~ms}$ & Amplitude de S: $0.05 \mathrm{mV}$ & FC média: $130 \mathrm{bpm}$ \\
\hline
\end{tabular}

\section{Comentários}

Avaliação eletrocardiográfica ritmo sinusal.

Onda P com duração e amplitude aumentada, sugestivo de sobrecarga atrial bilateral.

Onda QRS com duração aumentada e amplitude preservada, sugestivo de sobrecarga ventricular esquerda.

Intervalos PR e QT preservados.

Segmentos ST e onda T com amplitude preservados.

Eixo elétrico médio preservado.

Indicado realização de ecocardiograma.

Conclusões: Ritmo sinusal e sobrecarga atrial e ventricular esquerda.

A paciente continuou recebendo suporte de oxigenioterapia por meio do cateter nasal (Figura 5) durante o período de internação. Após a realização dos exames e confirmação do edema pulmonar cardiogênico decorrente de cardiopatia valvar esquerda, foram administrados Pimobendan na dose de $0,25 \mathrm{mg} / \mathrm{kg}$ de $12 / 12 \mathrm{~h}$ e Enalapril na dose de $0,3 \mathrm{mg} / \mathrm{kg}$ de $12 / 12 \mathrm{~h}$.

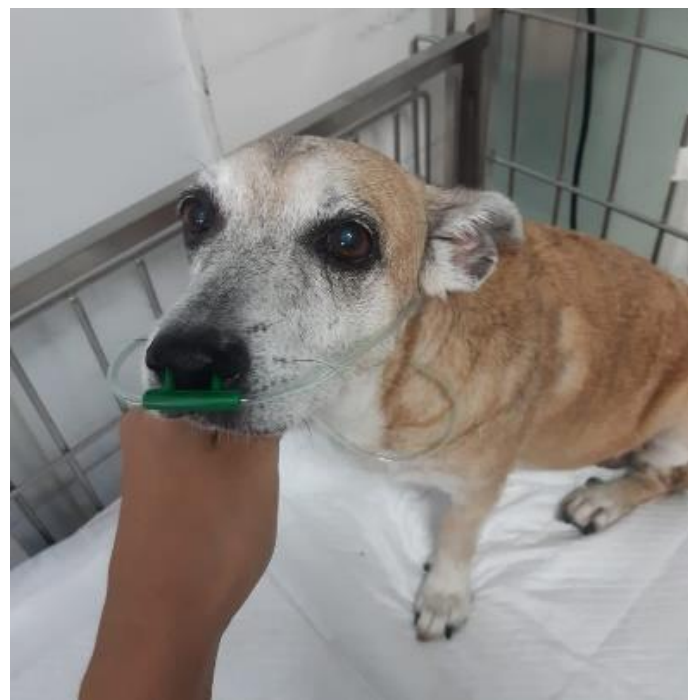

Figura 5. Paciente recebendo oxigenioterapia através do cateter nasal. 
Após o período de 24 horas de internação, a paciente encontrava-se estável, com parâmetros normais e sem a necessidade de oxigenioterapia. Recebeu alta médica e foram prescritos para continuar o tratamento em casa furosemida na dose de $3 \mathrm{mg} / \mathrm{kg}$ de $12 / 12$ horas durante 20 dias, pimobendan na dose de $0,25 \mathrm{mg} / \mathrm{kg}$ de $12 / 12$ horas para uso contínuo, enalapril na dose de $0,3 \mathrm{mg} / \mathrm{kg}$ de 12/12 horas para uso contínuo, prednisolona na dose de $0,5 \mathrm{mg} / \mathrm{kg}$ de $24 / 24$ horas durante 05 dias e ômega 1000mg de 24/24 horas durante 60 dias. Devido as medicações serem de uso contínuo, foi solicitado acompanhamento a cada 6 meses com cardiologista.

\section{Discussão}

O edema pulmonar pode levar o animal à óbito sendo necessário uma abordagem imediata. Corroborando com Bitencourt (2017) que considera uma urgência clínica que necessita de um rápido atendimento para estabilização do animal.

De acordo com Jericó et al. (2015) o edema pulmonar agudo em cães geralmente é resultado de afecção valvar degenerativa crônica, como a regurgitação mitral e a cardiomiopatia dilatada. Como foi relatado no caso, a paciente tinha uma cardiopatia esquerda, que foi confirmado pelos exames de radiografia e eletrocardiograma.

Conforme Bitencourt (2017) o edema pulmonar cardiogênico apresenta na ausculta cardíaca, ritmo de galope, sopro cardíaco e, dependendo da causa primária, arritmia. E também, segundo Jericó et al. (2015) os sinais clínicos variam de taquipneia a dispneia grave, similarmente aos sinais apresentados pela paciente ao adentrar na emergência da clínica.

No caso clínico, a abordagem inicial consistiu na estabilização do quadro do paciente por meio da oxigenioterapia e administração de diurético por via intravenosa. Jericó et al. (2015) afirmam que o paciente com congestão grave é, quase sempre, hemodinamicamente instável e um simples exame diagnóstico pode lhe ser fatal. Deve-se tratá-lo sintomaticamente para estabilização antes dos exames. O diagnóstico inicial é obtido por ausculta torácica e sintomas. De acordo com Bitencourt (2017) os diuréticos são utilizados na terapia do edema pulmonar, tanto cardiogênico como não cardiogênico, com o objetivo de reduzir o volume de fluido circulante e a pré-carga e, com isso, melhorar o edema pulmonar, sendo a furosemida, o diurético de escolha. Do mesmo modo foi usado no caso clínico a furosemida devido ao seu rápido início de ação.

O edema encontra-se normalmente na região dorso-caudal, bilateral e simétrico. O edema pulmonar cardiogênico é avaliado radiograficamente quanto à região cardíaca relativamente à silhueta cardíaca, aumento do átrio esquerdo e a dimensão dos vasos pulmonares (Castro, 2003; Viveiros, 2021). No caso clínico, os achados radiográficos consistiram nos lobos pulmonares caudais de padrão alveolar e tendendo à alveolar em lobo médio, com silhueta cardíaca de dimensões aumentadas em correspondência ao eixo ápicobasilar, promovendo desvio dorsal do trajeto traqueal, porém com grandes vasos de calibre preservados.

Segundo Viveiros (2021), a oxigenoterapia é o método utilizado na tentativa de contrariar os sinais de dispneia e cianose. E, o cateter nasal é uma técnica eficaz no aumento da concentração de oxigênio, útil para situações de oxigenoterapia por um longo período de tempo e para animais de grande porte que não caibam numa jaula de oxigênio. Assim como foi o método de escolha para fornecer oxigênio a paciente.

Os fármacos utilizados no tratamento crônico são a furosemida (dose: $1-2 \mathrm{mg} / \mathrm{kg}$ a cada $12-24 \mathrm{~h}$ $\mathrm{VO}$ ), os inibidores da enzima de conversão da angiotensina (IECAs) enalapril (dose: $0.25-0.5 \mathrm{mg} / \mathrm{kg} \mathrm{a}$ cada $24 \mathrm{~h} \mathrm{VO}$ ) e o inotrópico positivo pimobendan (dose:0.1-0.3 mg/kg a cada $12 \mathrm{~h}$ VO uma hora antes da alimentação). Para além da medicação devem ser realizadas alterações no estilo de vida do doente, promovendo um ambiente calmo e uma alimentação de baixo teor em sódio e adequada às necessidades calóricas, de modo a manter e/ou ganhar massa muscular magra. Corroborando com Viveiros (2021), no caso clínico foi prescrito enalapril, pimobendan e furosemida a paciente para tratamento crônico. 


\section{Conclusão}

O edema pulmonar cardiogênico se trata de uma emergência veterinária, onde primeiramente o paciente precisa ser estabilizado antes de realizar qualquer exame complementar e assim descobrir a causa primária. Após o diagnóstico, é necessário iniciar o tratamento com fármacos para controlar a cardiopatia paciente. Apesar de necessitar, na maioria das vezes, de uma abordagem emergencial, casos de edema pulmonar possuem prognóstico favorável quando iniciado o tratamento correto, pois as chances de reverter são altas, dependendo do quadro que o paciente chega na clínica e o tempo de resposta ao tratamento.

\section{Referências bibliográficas}

Bitencourt, E. H., Beier, S. L., \& Lima, M. P. A. (2017). Edema pulmonar agudo. Cadernos Técnicos de Veterinária e Zootecnia, 87, 9-17.

Bucceroni, T. C. (2021). Procedimento operacional padrão: manejo inicial do cão com dispneia. Universidade Federal de Uberlândia.

Castro, R. B. P. (2003). Edema pulmonar agudo. Medicina, 36(2/4), 200-204.

Ettinger, S. J., Fedlman, E. C., \& Taibo, R. A. (2002). Tratado de medicina interna veterinaria: enfermedades del perro y el gato. Manole.

Feldman, E. C. (1997). Tratado de medicina interna veterinária. In Moléstias do cão e do gato (Vol. 3).

Gaynor, J. S., \& Muir, W. W. (2009). Manual de controle da dor em medicina veterinária (Vol. 1). MedVet.

Glaus, T., Schellenberg, S., \& Lang, J. (2010). Cardiogenic and non cardiogenic pulmonary edema: pathomechanisms and causes. Schweizer Archiv Fur Tierheilkunde, 152(7), 311-317.

Irizarry, R. \& Reis, A. (2009). Beyond blood gases: Making uso of additional oxygenation parameters and plasma electrolytes in the emergency room. Compendium: Continuing Education for Veterinarians, 31, E1-E5.

Jericó, M. M., Kogika, M. M., \& Andrade Neto, J. P. (2015). Tratado de medicina interna de cães e gatos. Guanabara Koogan.

Rozanski, E. A., \& Rush, J. E. (2009). Manual colorido de medicina de urgência e terapia intensiva em pequenos animais. Artes Médicas.

Sumner, C., \& Rozanski, E. (2013). Management of respiratory emergencies in small animals. The Veterinary Clinics of North America. Small Animal Practice, 43(4), 799-815.

Viveiros, C. S. S. (2021). Edema pulmonar cardiogénico em felinos. Universidade de Évora - Escola de Ciências e Tecnologia. Évora, 2021.

Waddell, L. S. (2016). Oxygen Therapy. University Pensylvania, cliniciansbrief.com, p.43-48.

Histórico do artigo:

Recebido: 7 de julho de 2021

Aprovado: 18 de agosto de 2021
Licenciamento: Este artigo é publicado na modalidade Acesso Aberto sob a licença Creative Commons Atribuição 4.0 (CC-BY 4.0), a qual permite uso irrestrito, distribuição, reprodução em qualquer meio, desde que o autor e a fonte sejam devidamente creditados. 